\title{
Mass spectrometry-based metabolomics, analysis of metabolite-protein interactions, and imaging
}

\author{
Do Yup Lee ${ }^{1}$, Benjamin P. Bowen ${ }^{1}$, and Trent R. Northen ${ }^{1,2}$ \\ ${ }^{1}$ Department of GTL Bioenergy and Structural Biology, Life Sciences Division, Lawrence Berkeley National \\ Laboratory, Berkeley, CA, USA and ${ }^{2} J o i n t$ BioEnergy Institute (JBEI), Emeryville, CA, USA
}

BioTechniques 49:557-565 (August 2010) doi 10.2144/000113451

Keywords: mass spectrometry; metabolomics; functional genomics; enzyme assays

Our understanding of biology has been greatly improved through recent developments in mass spectrometry, which is providing detailed information on protein and metabolite composition as well as protein-metabolite interactions. The high sensitivity and resolution of mass spectrometry achieved with liquid or gas chromatography allows for detection and quantification of hundreds to thousands of molecules in a single measurement. Where homogenization-based sample preparation and extraction methods result in a loss of spatial information, mass spectrometry imaging technologies provide the in situ distribution profiles of metabolites and proteins within tissues. Mass spectrometry-based analysis of metabolite abundance, protein-metabolite interactions, and spatial distribution of compounds facilitates the high-throughput screening of biochemical reactions, the reconstruction of metabolic networks, biomarker discovery, determination of tissue compositions, and functional annotation of both proteins and metabolites.

Mass spectrometry (MS) is a rapidly growing technology for the comprehensive profiling of small molecules (metabolites) and proteins $(1,2)$. Information obtained from the metabolome is particularly useful given that small molecules represent the downstream outcome of cellular machinery (i.e., enzymes) and can provide a metabolic phenotype of a biological system. Another advantage of studying the metabolome (versus the proteome or genome) is that the analysis can be performed independently of a genome sequence or large expressed sequence tag databases and therefore can be applied to virtually any biological system.

In most cases, the first step in a metabolite profiling experiment is to extract metabolites from the biological matrix (Figure 1). Extracted metabolites are often separated using gas chromatography/MS (GC/MS) (3), liquid chromatography/MS (LC/MS) (4), or capillary electrophoresis/MS (CE/MS) (5). Critical to all MS-based approaches is the efficient desorption and ionization of metabolites, where the resulting gas phase ions can be separated by mass analyzers such as quadrupole, time-of-flight, and ion trap. Ions are typically detected using a microchannel plate and photomultiplier tube and identified through comparison of exact mass, retention time, and fragmentation information with genuine standards and spectral databases. This review introduces general concepts and technical approaches to metabolite profiling, characterization of metabolite-protein interactions, and metabolite imaging.

\section{Sample preparation}

Sample preparation is critical to metabolite analysis. While methods vary depending on the experimental goal, they are typically divided into the following steps: (i) quenching to halt metabolism, (ii) cell harvesting through medium removal (in the case of microorganism and animal cell cultures), (iii) cell lysis, and (iv) metabolite extraction (6). Commonly, cellular metabolism is quenched through the addition of cold methanol to a culture broth or flash-freezing of tissues in liquid nitrogen (7). This procedure can be skipped by utilizing a filtration method that enables fast separation of cells from the medium (8). For metabolite extraction, a wide range of solvent systems and temperatures are used (from boiling ethanol to cold mixtures of organic solvents); however, methods of choice include cold methanol and mixtures of methanol and chloroform (9-13).

\section{Ion source technology}

MS requires formation of gas phase ions that can be resolved through the manip- ulation of electromagnetic fields. This is commonly achieved using electron impact ionization (EI), electrospray ionization (ESI), or matrix-assisted laser desorption/ ionization (MALDI); more detail can be found in Siuzdak's excellent text (14). Briefly, in EI, volatile metabolites are first separated using GC and then ionized by bombardment with an electron beam; this process results in formation of radical cations and extensive fragmentation. Many metabolites have insufficient vapor pressure, even at high temperature (i.e., sugars, amino acids), and must be chemically modified (derivatized typically via silylation or alkylation) to increase their volatility and stability. However, chemical modification is not well-suited for thermally labile molecules or for chemicals lacking derivatizable groups (e.g., amino, hydroxyl, or carboxyl groups). Lee and Fiehn's article offers detailed discussion and protocols for GC/MS metabolite analysis (9).

The development of ESI has been a breakthrough for MS analysis of intact biomolecules. The technique allows desorption and ionization of a wide range of molecules directly from the liquid phase. Therefore, it can be directly interfaced with either LC or CE.ESI is based on the formation and drying of charged liquid droplets. Fine droplets are formed through a charged nebulizer needle. As solvent evaporates, the charges are concen- 

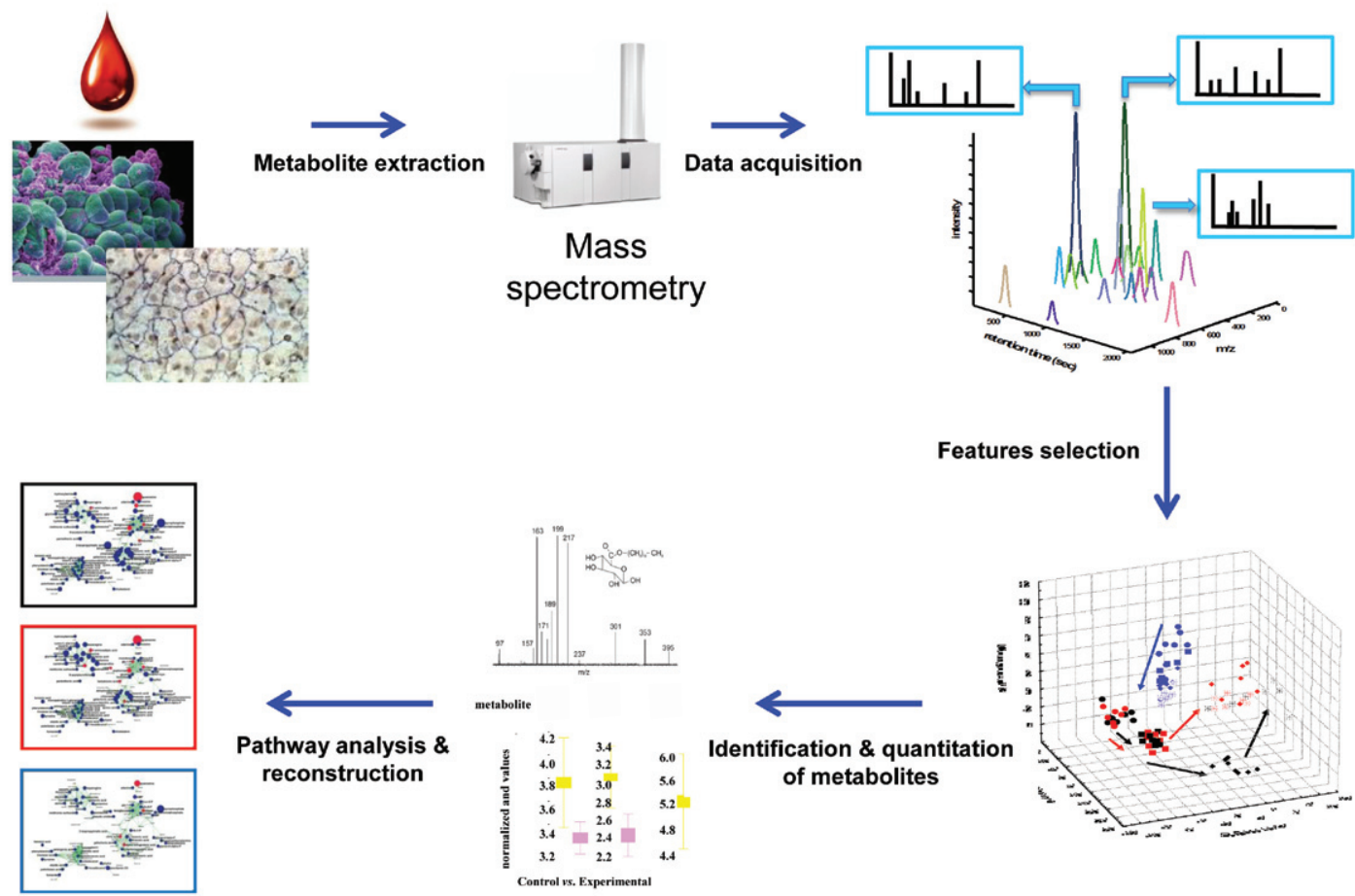

Figure 1. Conventional untargeted metabolomics workflow. Small-molecule metabolites are extracted from the sample matrix. Metabolites are separated using chromatographic steps, ionized, and analyzed using MS. Features of interest are selected from raw data using univariate and multivariate statistical approaches. Features are then identified using database searches, comparisons to authentic standards, and MS/MS. Identified features can then be used for reaction monitoring, pathway analysis, and metabolic network reconstruction.

trated on the surface. When surface charge repulsion exceeds surface tension, droplets break up into smaller droplets. This consecutive concentration process continues until gas phase ions are ultimately formed.

MALDI is another widely used ionization approach for biomolecules. MALDI is performed using a large excess of compounds (matrix) that absorb light and sublime into the gas phase. These matrices efficiently assist desorption and ionization of a broad range of analytes for subsequent mass spectrometric analysis. However, the matrix itself ionizes and is detected, which greatly complicates analysis for ions with mass-to-charge ratio $(m / z)<500 \mathrm{Da}$. It should be noted that the composition of the MALDI matrix can be modified to reduce these matrix background effects (15-17), and there have been recent innovations in matrix-free technologies. For example, nanostructure-initiator MS (NIMS) is a new technology for metabolite analysis. Here, metabolites are adsorbed onto a vacuum-compatible initiator liquid-coated nanostructured surface (18). This surface, when irradiated with a laser, vaporizes the initiator causing the desorption and ionization of analytes. It should be noted that the adsorption of nonpolar metabolites to a hydrophobic NIMS surfaces has been found to reduce signal suppression in complex biological samples (18).

\section{Tandem MS}

Fragmention experiments [tandem MS $\left(\mathrm{MS} / \mathrm{MS}\right.$ or $\left.\mathrm{MS}^{\mathrm{n}}\right)$ ] are important to biomol- ecule analysis and enable the comparison of experimental fragmentation patterns with authentic standards and spectral databases to confirm molecular structure. The existence of metabolite spectral databases, many of which include fragmentation patterns and retention time indices, facilitates identification using GC/MS and LC/MS/MS [i.e., Golm library, Fiehn library, National Institute of Standards and Technology (NIST), Metlin, and MassBank] (19). In cases where metabolites are not in such databases, a knowledge of the precursor (unfragmented) ion's exact mass, the exact mass of the fragment ions, and their corresponding isotopic distributions is useful for metabolite identification (20).

Fragmentation information can be acquired through various combinations of mass analyzers to isolate and fragment target ions and to analyze/detect the resulting fragments. Some of the most commonly used tandem mass spectrometers include quadrupole time-of-flight (Q-TOF), triple quadrupole (QQQ), ion trap, and Fourier transform ion cyclotron resonance (FT-ICR). Q-TOF MS instruments have high resolution, mass accuracy, and scan rate, enabling MS profiling and MS/MS analysis within a single experiment. In this case, the first quadrupole isolates ions of interest, which are then fragmented in a collision cell and analyzed by a TOF mass analyzer. QQQ mass analyzers possess three quadrupoles in a row. The first isolates, the second fragments, and the third analyzes the ions. This makes targeted MS analysis of precursor ions and MS/MS analysis of metabolites by product ion monitoring possible. This instrument can also be used for selected reaction monitoring (SRM), which provides excellent sensitivity and quantitation. Linear ion trap mass analyzers are commonly used for analysis of low abundance compounds due to their high trapping capacity as well as in $\mathrm{MS}^{\mathrm{n}}$ experiments to gain additional structural information through repeated rounds of fragmentation $(21,22)$. While linear ion traps have low mass accuracy, FT-ICR-MS offers the highest level of resolution $(>1,000,000)$, excellent mass accuracy (typically $\sim 1 \mathrm{ppm}$ ), and good dynamic range $(\sim 5000)(23)$. The related orbitrap mass spectrometer has been another major breakthrough. It provides comparable mass accuracy (2-5 ppm), mass resolution $(150,000)$, and $\mathrm{m} / \mathrm{z}$ range of 6000 to FT-ICR-MS, but has a considerably faster scan rate. The orbitrap and FT-ICR mass spectrometers are usually operated as a hybrid instrument with a linear ion trap (24).

\section{Targeted and global metabolomics}

Metabolomic approaches are often divided into targeted and untargeted. As the name suggests, targeted methods (25) are designed to detect and often quantify specific metabolites of interest within a sample. This approach has the advantage 
A

Protein-ligand reaction
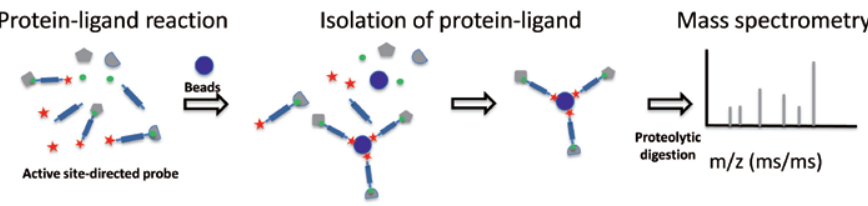

B

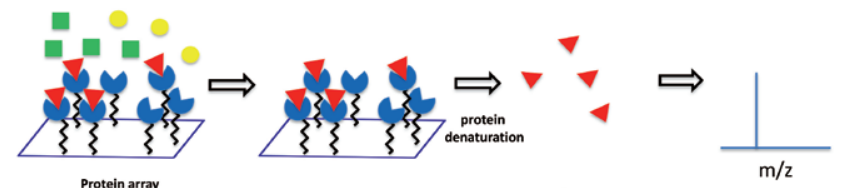

C

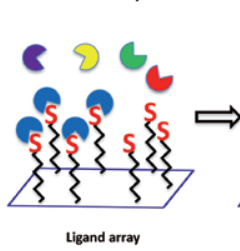

Q Proteins
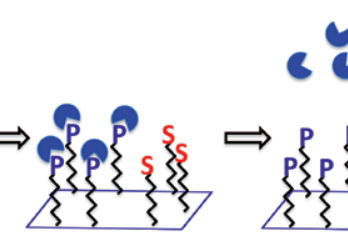
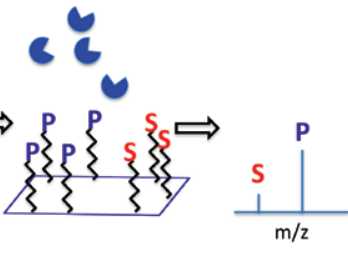

$\mathrm{m} / \mathrm{z}$

Figure 2. MS analysis of protein-metabolite interaction. (A) ABPP utilizes chemical probes and beads to selectively profile actively bound proteins through specific crosslinking reactions and proteomic analysis of tagged peptides. (B) Protein arrays are used to bind ligands that can subsequently be released for MS-based identification. (C) Surface-immobilized metabolites can bind proteins (proteomic analysis) or detect specific enzymatic transformations of the immobilized substrate.

of maximizing the specificity and the sensitivity of MS methods. Consequently, the targeted analyses utilize analytical standards to define suitable GC or LC methods, determine metabolite fragmentation patterns, and construct calibration curves for absolute quantification.

Clinical diagnostics is an early example of targeted metabolomics (26), where methods were developed to measure amino acids, drug metabolites, and specific endogenous compounds. Other targeted approaches include measurement of enzymatic activities in vitro (27) and simultaneous monitoring of multiple glycosylhydrolases and glycosyltransferases (28). Targeted methods have also been used for pharmaceutical development, the validation of enzymatic activities for putative enzymes $(29,30)$, and for the identification of specific substrates for putative enzymes $(28,31)$.

In contrast, untargeted global metabolite profiling aims to maximize coverage of metabolites, often compromising the sensitivity and specificity for any particular metabolite. These metabolomic approaches involve less up-front method development when compared with targeted approaches, but require much more data analysis. The overall metabolomic workflow for untargeted LC/MS is summarized in Figure 1, and the reader is referred to detailed protocols to assist in implementation (32). In a metabolomics experiment, sample preparation, chromatographic conditions (33), and MS ionization are all optimized to maximize the diversity of metabolites detected (34). Interpretation of the hundreds or thousands of resulting ions is challenging due to a large number of unknowns, and their identification is further complicated by the many experimental artifacts (i.e., adducts, neutral losses, isotopes). Hence, analysis depends extensively on computational tools, statistical methods, and metabolite databases.

The first step of untargeted metabolomic data analysis is to define features: the combination of the exact $m / z$ and the corresponding LC retention time (RT). These $m / z \times$ RT dimensions subsequently are used as initial metabolite identifiers that can be quantitatively compared with important features for further analysis. There is a wide range of algorithms for identification and comparison of features, as recently reviewed by De Vos et al. (32). The most widely used algorithms are XCMS (35), msInspect (36), and mzMine (37). Identified features are quantitatively compared using univariate and multivariate statistics to select the most important features for final identification [e.g., principle component analysis (PCA) (38), partial least-squares discriminant analysis (PLS-DA) (39), and self-organizing networks (40)].

Subsequent feature identification relies primarily on exact mass searches against metabolite databases such as KEGG (41), Metlin (19), Golm (42), and HMDB (43). Database searching often results in multiple identifications for a particular ion due to insufficient mass accuracy and/or degenerate empirical formulas for a given exact mass (i.e., isomers). In these cases, identification requires analytical standards to further define the molecule's retention time and fragmentation pattern. Unfortunately, only a relatively small subset of metabolites is commercially available. Thus, identification of unknown ions often requires either preparative-scale HPLC to enrich for NMR studies or chemical synthesis to compare with unknowns using MS/MS.

Several recent advances in the application of LC/MS and GC/MS have simplified the identification procedure: stable isotopelabeled metabolites are often used to trace pathways within metabolic networks (44). The resulting shift in $\mathrm{m} / \mathrm{z}$ for the heavier metabolites is used to specify the labeling position (45), to discriminate between alternative pathways, and to estimate metabolic flux (46). In a recent example, metabolic transformations of the antiretroviral drug indinavir in vivo have been studied using knowledge-based predictions coupled with subsequent $\mathrm{LC} / \mathrm{MS} / \mathrm{MS}$ analysis (47). This study identified 18 metabolites of indinavir after incubation of the drug with human hepatic postmitochondrial preparations.

In GC/MS studies, classification and annotation of unidentified features has been improved through extraction of substructural information to predict target classes (48). Decision tree (DT)-based prediction algorithm makes use of mass spectral fragmentation and retention index (RI) information to enable sensitive and precise detection of compound substructures. It has also been shown that higher intensities of molecular and total ions ( $\leq 10$-fold) are achieved by modulating a beam-steering voltage of the ion source at $70 \mathrm{eV}$ EI of GC-TOF (e.g., alkanes, fatty acid methyl esters, and trimethylsilylated metabolites) (49). In this application, the accurate masses and isotopic data allowed more precise calculations of elemental compositions and facilitated metabolite identification when combined with fragmentation patterns from EI data.

\section{MS analysis of metabolite- protein interaction}

MS is being used for annotation of putative enzyme functions, suggesting potential metabolic reactions, and validating the existence of metabolic pathways using in vitro enzymatic assays. Such studies can be broken down into three groups, as shown in Figure 2.

\section{Solution-based in vitro \\ binding or enzyme assays}

This is a metabolomics profiling experiment that examines the loss or appearance of metabolites following enzymatic reactions. A limitation of this approach is that these metabolites often need to be detected within a complex sample matrix (i.e., in a cell lysate). Recently, this approach 


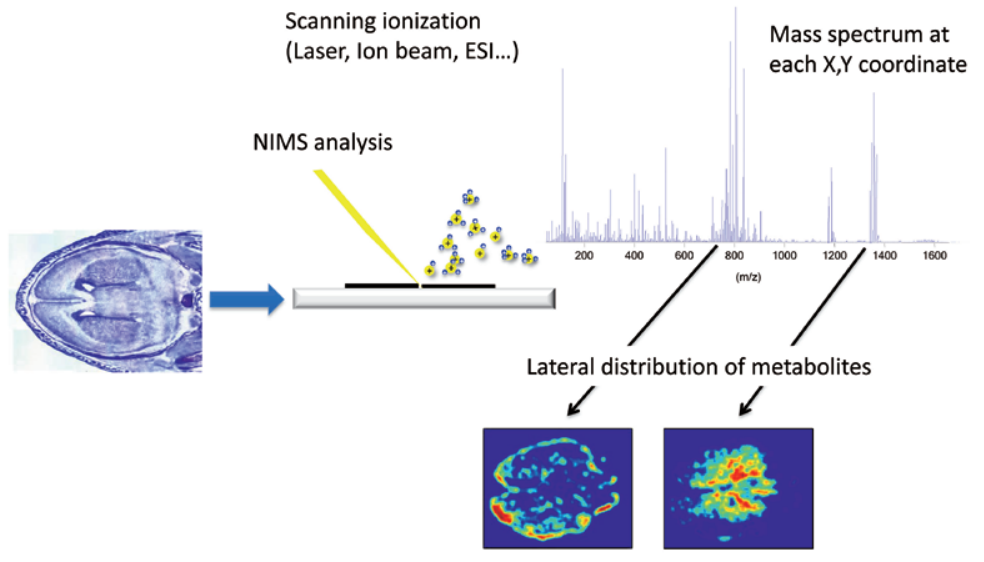

Figure 3. MS imaging approaches are used to study the spatial distribution of metabolites within biological samples. Metabolites are desorbed and ionized, and mass spectra are collected. The resulting data enables image reconstruction that can be used to define metabolite localization patterns.

was used to discover specific substrates (several important lipid species) that were enzymatically hydrolyzed by fatty acid amide hydrolase (FAAH) in vivo (50). Another example of this approach is the genome-wide analysis of protein activity and substrate selectivity using activitybased protein profiling (ABPP) $(51,52)$. Activity and selectivity estimates can be made using this tag-free ABPP strategy (click chemistry) $(53,54)$.

\section{Immobilized protein-based assays}

Here a protein is immobilized onto a surface or particle (i.e., Sephadex) and used to study interactions with small molecules (ligands). As an early example, high-throughput screening of inhibitors was done using immobilized enzyme, resulting in the identification of 17 pepsin and 6 glutathione $S$-transferase inhibitors (55). In a more recent example, an immobilized protein assay was applied to identify specific protein interactions with multiple metabolites from cellular extracts (56).

\section{Immobilized metabolite}

\section{binding/enzyme assays}

Here, one or more metabolites are immobilized and used as bait to identify their interacting protein partner(s) either by physically isolating the protein or by using affinity chromatography. This approach was used to establish 11 interaction partners with the new metabolite $N^{4}$ - $(N$-acetylaminopropyl) spermidine by immobilizing the metabolite on agarose beads, binding the protein(s), and elution for subsequent proteomic identification (57).

Another recent example is the Nimzyme assay, which uses fluorous phase interactions for soft immobilization of the enzyme substrates onto a NIMS (58). This approach allows direct characterization of crude cell extracts, since the immobilized substrates and products are retained on the surface. It has been shown to be suitable for detection of both addition and cleavage reactions (sialyltransferase and galactosidase). In a related approach, alkanethiol monolayers are used for substrate immobilization on metal surfaces, and laser desorption ionization MS is used to analyze reaction products following enzyme treatments (59).

\section{MS-based metabolite imaging}

A limitation of LC/MS, CE/MS, or GC/MS methods is the loss of spatial information that results upon metabolite extraction from homogenized samples. Metabolomic imaging technologies, therefore, can be an important alternative and provide information on the spatial distribution of metabolites within tissues (Figure 3). MALDI imaging is the most widely used (60) MS-based tissue imaging approach. MALDI matrix is typically applied to the sample (i.e., tissue) either by spotting or spraying, and images are generated by raster scanning the laser over the sample, providing a mass spectrum at each $x, y$ coordinate. Composite images are constructed by mapping the distribution and abundance of ions within the sample.

Another widely used approach for imaging is secondary ion MS (SIMS) (61). In SIMS, the sample is sputtered with ions (i.e., gold, gallium, bismuth) to generate secondary ions. The resultant ions are analyzed typically using time-of-flight mass analyzers (TOF-SIMS). This matrix-free technique does not have the background matrix ions and also has the advantage that ion beams can be focused to $\sim 100 \mathrm{~nm}$. However, the energetic SIMS ionization process results in extensive molecular fragmentation that significantly complicates metabolite identification and data interpretation.
Such limitations in imaging analysis are now being addressed using new soft techniques, such as desorption electrospray ionization (DESI). DESI is based on scanning the surface with the electrospray cone (62). NIMS is another matrixfree approach that uses a scanning laser system to generate ions from the liquidfilled nanostructured surface (18).

\section{Conclusion}

The application of MS to metabolite analysis is providing new insights into the biochemical functions and the cellular physiology of living organisms. These approaches are becoming widely used for biomarker discovery and systematic investigation of metabolic dynamics. Targeted approaches are used to monitor and often quantify specific metabolites of interest with high sensitivity and selectivity, whereas untargeted metabolomic profiling is allowing detection of unexpected metabolites. Specific reactions can be probed using activity-based metabolite/protein profiling and MS-based enzyme assays, and now developments in MS-based imaging are enabling the determination of metabolite spatial distribution within tissues.

\section{Acknowledgements}

We gratefully acknowledge support from the Department of Energy (DE-AC02-05CH11231), the National Institutes of Health $(\mathrm{NIH})$, the National Cancer Institute (NCI), Bay Area Breast Cancer SPORE (P50 CA 58207), and the California Breast Cancer Research Program (15IB-0063). We also thank Deron Herr at Scripps Research Institute images. This paper is subject to the NIH Public Access Policy.

\section{Competing interests}

The authors declare no competing interests.

\section{References}

1. Fiehn, O.2002. Metabolomics-the link between genotypes and phenotypes. Plant Mol. Biol. 48:155-171.

2. Nielsen, J. and S. Oliver. 2005. The next wave in metabolome analysis. Trends Biotechnol. 23:544-546.

3. Fiehn, O., J. Kopka, P. Dörmann, T. Altmann, R.N. Trethewey, and L. Willmitzer. 2000. Metabolite profiling for plant functional genomics. Nat. Biotechnol. 18:1157-1161.

4. Plumb, R.S., C.L. Stumpf, M.V. Gorenstein, J.M. Castro-Perez, G.J. Dear, M. Anthony, B.C. Sweatman, S.C. Connor, and J.N. Haselden. 2002. Metabonomics: the use of electrospray 
mass spectrometry coupled to reversed-phase liquid chromatography shows potential for the screening of rat urine in drug development. Rapid Commun. Mass Spectrom. 16:1991-1996.

5. Soga, T., Y. Ohashi, Y. Ueno, H. Naraoka, M. Tomita, and T. Nishioka. 2003. Quantitative metabolome analysis using capillary electrophoresis mass spectrometry. J. Proteome Res. 2:488-494.

6. Villas-Boas, S.G., J. Hojer-Pedersen, M. Akesson, J. Smedsgaard, and J. Nielsen. 2005. Global metabolite analysis of yeast: evaluation of sample preparation methods. Yeast 22:1155-1169.

7. Kopka, J., A. Fernie, W. Weckwerth, Y. Gibon, and M. Stitt. 2004. Metabolite profiling in plant biology: platforms and destinations. Genome Biol. 5:109.

8. Bolten, C.J., P. Kiefer, F. Letisse, J.C. Portais, and C. Wittmann. 2007. Sampling for metabolome analysis of microorganisms. Anal. Chem. 79:38433849 .

9. Lee, D.Y. and O. Fiehn. 2008. High quality metabolomic data for Chlamydomonas reinhardtii. Plant Methods 4:7.

10. Want,E.J.,G.O'Maille,C.A.Smith,T.R.Brandon,W.Uritboonthai,C.Qin,S.A. Trauger, and G. Siuzdak. 2006. Solvent-dependent metabolite distribution, clustering, and protein extraction for serum profiling with mass spectrometry. Anal. Chem. 78:743-752.

11. Gullberg, J., P.Jonsson, A. Nordström, M.Sjöström, and T. Moritz. 2004. Design of experiments: an efficient strategy to identify factors influencing extraction and derivatization of Arabidopsis thaliana samples in metabolomic studies with gas chromatography/mass spectrometry. Anal. Biochem. 331:283-295.

12. Koek, M.M., B. Muilwijk, M.J. van der Werf, and T. Hankemeier. 2006. Microbial metabolomics with gas chromatography/mass spectrometry. Anal. Chem. 78:1272-1281.

13. Lisec, J., N. Schauer, J. Kopka, L. Willmitzer, and A.R. Fernie. 2006. Gas chromatography mass spectrometry-based metabolite profiling in plants. Nat. Protocols 1:387-396.

14. Siuzdak, G. 1996. Mass Spectrometry for Biotechnology. Academic Press, San Diego.

15. Guo, Z., Q. Zhang, H. Zou, B. Guo, and J. Ni. 2002. A method for the analysis of low-mass molecules by MALDI-TOF mass spectrometry. Anal. Chem. 74:1637-1641.

16. McCombie, G. and R. Knochenmuss. 2004. Small-molecule MALDI using the matrix suppression effect to reduce or eliminate matrix background interferences. Anal. Chem. 76:4990-4997.

\section{BioTechnioues}

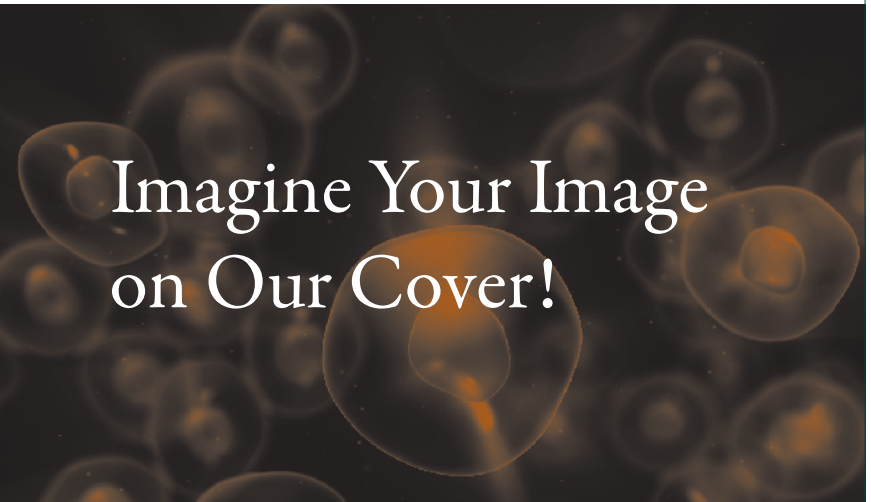

Did you photograph something truly unique under the microscope today? Do you have a stunning image that conveys a critical scientific concept with aesthetic appeal?

We are looking for your images to display on the cover of BioTechniques.

Please submit any potential cover images along with your manuscript submission at www.editorialmanager.com/bt

Cover images submitted independently of a manuscript can be emailed to bioeditor@biotechniques.com
17. Reyzer, M.L., Y. Hsieh, K. Ng, W.A. Korfmacher, and R.M. Caprioli. 2003. Direct analysis of drug candidates in tissue by matrix-assisted laser desorption/ ionization mass spectrometry. J. Mass Spectrom. 38:1081-1092.

18. Northen, T.R., O. Yanes, M.T. Northen, D. Marrinucci, W. Uritboonthai, J. Apon, S.L. Golledge, A. Nordström, and G. Siuzdak. 2007. Clathrate nanostructures for mass spectrometry. Nature 449:1033-1036.

19. Smith, C.A., G. O’Maille, E.J. Want, C. Qin, S.A. Trauger, T.R. Brandon, D.E. Custodio, R. Abagyan, and G. Siuzdak. 2005. METLIN-A metabolite mass spectral database. Ther. Drug Monit. 27:747-751.

20. Kind, T. and O. Fiehn. 2007. Seven golden rules for heuristic filtering of molecular formulas obtained by accurate mass spectrometry. BMC Bioinformatics $8: 105$.

21. Allwood, J.W. and R. Goodacre. 2009. An introduction to liquid chromatography-mass spectrometry instrumentation applied in plant metabolomic analyses. Phytochem. Anal. 21:33-47.

22. Balogh, M.P. 2004. Debating resolution and mass accuracy. LCGC North America 22:118-131.

23. Barrow, M.P., W.I. Burkitt, and P.J. Derrick. 2005. Principles of Fourier transform ion cyclotron resonance mass spectrometry and its application in structural biology. Analyst 130:18-28.

24. Hu, Q., R.J. Noll, H. Li, A. Makarov, M. Hardman, and R. Graham Cooks. 2005. The Orbitrap: a new mass spectrometer. J. Mass Spectrom. 40:430-443

25. Bennett, B.D., J. Yuan, E.H. Kimball, and J.D. Rabinowitz. 2008. Absolute quantitation of intracellular metabolite concentrations by an isotope ratiobased approach. Nat. Protocols 3:1299-1311.

26. Chace, D.H., D.S. Millington, N. Terada, S.G. Kahler, C.R. Roe, and L.F. Hofman. 1993. Rapid diagnosis of phenylketonuria by quantitative analysis for phenylalanine and tyrosine in neonatal blood spots by tandem mass spectrometry. Clin. Chem. 39:66-71.

27. Liesener, A. and U. Karst. 2005. Monitoring enzymatic conversions by mass spectrometry: a critical review. Anal. Bioanal. Chem. 382:1451-1464.

28. Yu, Y., K. Ko, C. Zea, and N. Pohl. 2004. Discovery of the chemical function of glycosidases: design, synthesis, and evaluation of mass-differentiated carbohydrate libraries. Org. Lett. 6:2031-2033.

29. Lin, H., M.A. Fischbach, D.R. Liu, and C.T. Walsh. 2005. In vitro characterization of salmochelin and enterobactin trilactone hydrolases IroD, IroE, and Fes. J. Am. Chem. Soc. 127:11075-11084.

30. Fischbach, M.A., H. Lin, D.R. Liu, and C.T. Walsh. 2005. In vitro characterization of IroB, a pathogen-associated C-glycosyltransferase. Proc. Natl. Acad. Sci. USA 102:571-576.

31. Furuya, T., T. Nishi, D. Shibata, H. Suzuki, D. Ohta, and K. Kino. 2008. Characterization of orphan monooxygenases by rapid substrate screening using FT-ICR mass spectrometry. Chem. Biol. 15:563-572.

32. De Vos, R.C.H., S. Moco, A. Lommen, J.J.B. Keurentjes, R.J. Bino, and R.D. Hall. 2007. Untargeted large-scale plant metabolomics using liquid chromatography coupled to mass spectrometry. Nat. Protocols 2:778-791.

33. Garcia, D.E., E.E. Baidoo, P.I. Benke, F. Pingitore, Y.J. Tang, S. Villa, and J.D. Keasling. 2008. Separation and mass spectrometry in microbial metabolomics. Curr. Opin. Microbiol. 11:233-239.

34. Nordstrom, A., E. Want, T. Northen, J. Lehtio, and G. Siuzdak. 2008. Multiple ionization mass spectrometry strategy used to reveal the complexity of metabolomics. Anal. Chem. 80:421-429.

35. Smith, C.A., E.J. Want, G. O'Maille, R.Abagyan, and G.Siuzdak. 2006.XCMS: processing mass spectrometry data for metabolite profiling using nonlinear peak alignment, matching, and identification. Anal. Chem. 78:779-787.

36. Bellew, M., M. Coram, M. Fitzgibbon, M. Igra, T. Randolph, P Wang, D. May, J. Eng, et al. 2006. A suite of algorithms for the comprehensive analysis of complex protein mixtures using high-resolution LC-MS. Bioinformatics 22:1902-1909.

37. Katajamaa, M. and M. Orešič. 2007. Data processing for mass spectrometrybased metabolomics. J. Chromatogr. A 1158:318-328.

38.Taylor, J., R.D. King, T. ltmann, and O. Fiehn. 2002. Application of metabolomics to plant genotype discrimination using statistics and machine learning. Bioinformatics 18:S241-S248.

39. Jansson, J., B. Willing, M. Lucio, A. Fekete, J. Dicksved, J. Halfvarson, C. Tysk, and P.Schmitt-Kopplin. 2009. Metabolomics reveals metabolic biomarkers of Crohn's disease. PLoS One 4:e6386.

40. Patterson, A.D., H. Li, G.S. Eichler, K.W. Krausz, J.N. Weinstein, A.J. Fornace, Jr., F.J. Gonzalez, and J.R. Idle. 2008. UPLC-ESI-TOFMSbased metabolomics and gene expression dynamics inspector self-organizing metabolomic maps as tools for understanding the cellular response to ionizing radiation. Anal. Chem. 80:665-674.

41. Kanehisa, M. and S. Goto. 2000. KEGG: kyoto encyclopedia of genes and genomes. Nucleic Acids Res. 28:27-30. 
42. Kopka, J., N. Schauer, S. Krueger, C. Birkemeyer, B. Usadel, E. Bergmuller, P. Dormann, W. Weckwerth, et al.2005.GMD@CSB.DB: the Golm Metabolome Database. Bioinformatics 21:1635-1638.

43. Wishart, D.S., D. Tzur, C. Knox, R. Eisner, A.C. Guo, N. Young, D. Cheng, K. Jewell, et al. 2007. HMDB: the human metabolome database. Nucleic Acids Res. 35:D521-D526.

44. Droste, P., M. Weitzel, and W. Wiechert. 2008. Visual exploration of isotope labeling networks in 3D. Bioprocess Biosyst. Eng. 31:227239.

45. Tang, Y., F. Pingitore, A. Mukhopadhyay, R. Phan, T.C. Hazen, and J.D. Keasling. 2007 Pathway confirmation and flux analysis of central metabolic pathways in Desulfovibrio vulgaris hildenborough using gas chromatography-mass spectrometry and Fourier transform-ion cyclotron resonance mass spectrometry. J. Bacteriol. 189:940-949.

46. Sauer, U. 2006. Metabolic networks in motion: ${ }^{13} \mathrm{C}$-based flux analysis. Mol. Syst. Biol. 2:62.

47. Anari, M.R., R.I. Sanchez, R. Bakhtiar, R.B. Franklin, and T.A. Baillie. 2004. Integration of knowledge-based metabolic predictions with liquid chromatography data-dependent tandem mass spectrometry for drug metabolism studies: application to studies on the biotransformation of indinavir. Anal. Chem. 76:823-832.

48.Hummel, J., N. Strehmel, J. Selbig, D. Walther, and J. Kopka. 2010. Decision tree supported substructure prediction of metabolites from GC-MS profiles. Metabolomics $6: 322-333$.
49. Abate, S., Y.G. Ahn, T. Kind, T.R.I. Cataldi, and O. Fiehn. 2010. Determination of elemental compositions by gas chromatography/time-offlight mass spectrometry using chemical and electron ionization. Rapid Commun. Mass Spectrom. 24:1172-1180.

50. Saghatelian, A., S.A. Trauger, E.J. Want, E.G. Hawkins, G. Siuzdak, and B.F. Cravatt. 2004. Assignment of endogenous substrates to enzymes by global metabolite profiling. Biochemistry 43:14332-14339.

51. Kidd, D., Y. Liu, and B.F. Cravatt. 2001. Profiling serine hydrolase activities in complex proteomes. Biochemistry 40:4005-4015.

52. Speers, A.E. and B.F. Cravatt. 2004. Profiling enzyme activities in vivo using click chemistry methods. Chem. Biol. 11:535-546.

53. Jessani, N., Y. Liu, M. Humphrey, and B.F. Cravatt. 2002. Enzyme activity profiles of the secreted and membrane proteome that depict cancer cell invasiveness. Proc. Natl. Acad. Sci. USA 99:10335-10340.

54. Speers, A.E., G.C. Adam, and B.F. Cravatt. 2003. Activity-based protein profiling in vivo using a copper(I)-catalyzed azide-alkyne [3 + 2] cycloaddition. J. Am. Chem. Soc. 125:4686-4687.

55. Cancilla, M.T., M.D. Leavell, J. Chow, and J.A. Leary. 2000. Mass spectrometry and immobilized enzymes for the screening of inhibitor libraries. Proc. Natl. Acad. Sci. USA 97:12008-12013.

56. Morozov, V.N., T.Y. Morozova, K.L. Johnson, and S. Naylor. 2003. Parallel determination of multiple protein metabolite interactions using cell extract, protein microarrays and mass spectrometric detection. Rapid Commun. Mass Spectrom. 17:2430-2438.
57. Kalisiak, J., S.A. Trauger, E. Kalisiak, H. Morita, V.V. Fokin, M.W.W.Adams, K.B. Sharpless, and G. Siuzdak. 2009. Identification of a new endogenous metabolite and the characterization of its protein interactions through an immobilization approach. J. Am. Chem. Soc. 131:378-386.

58. Northen, T.R., J.C. Lee, L. Hoang, J. Raymond, D.R. Hwang, S.M. Yannone, C.H. Wong, and G. Siuzdak. 2008. A nanostructure-initiator mass spectrometry-based enzyme activity assay. Proc. Natl. Acad. Sci. USA 105:3678-3683.

59. Ban, L. and M. Mrksich. 2008. On-chip synthesis and label-free assays of oligosaccharide arrays. Angew. Chem. Int. Ed. Engl. 47:3396-3399.

60. Cornett, D.S., M.L. Reyzer, P. Chaurand, and R.M. Caprioli. 2007. MALDI imaging mass spectrometry: molecular snapshots of biochemical systems. Nat. Methods 4:828-833.

61. Winograd, N. 2005. The Magic of Cluster SIMS. Anal. Chem. 77:142A-149A.

62. Wiseman, J.M., D.R. Ifa, Q. Song, and R.G. Cooks. 2006. Tissue imaging at atmospheric pressure using desorption electrospray ionization (DESI) mass spectrometry. Angew. Chem. Int. Ed. Engl. 45:7188-7192.

Received 22 April 2010; accepted 24 June 2010.

Address correspondence to Trent R. Northen, Department of GTL Bioenergy and Structural Biology, Life Sciences Division, Lawrence Berkeley National Laboratory, 1 Cyclotron Road, Berkeley, CA 94720, USA. e-mail: trnorthen@lbl.gov
New Microcalorimetry Products

\section{From TA Instruments}

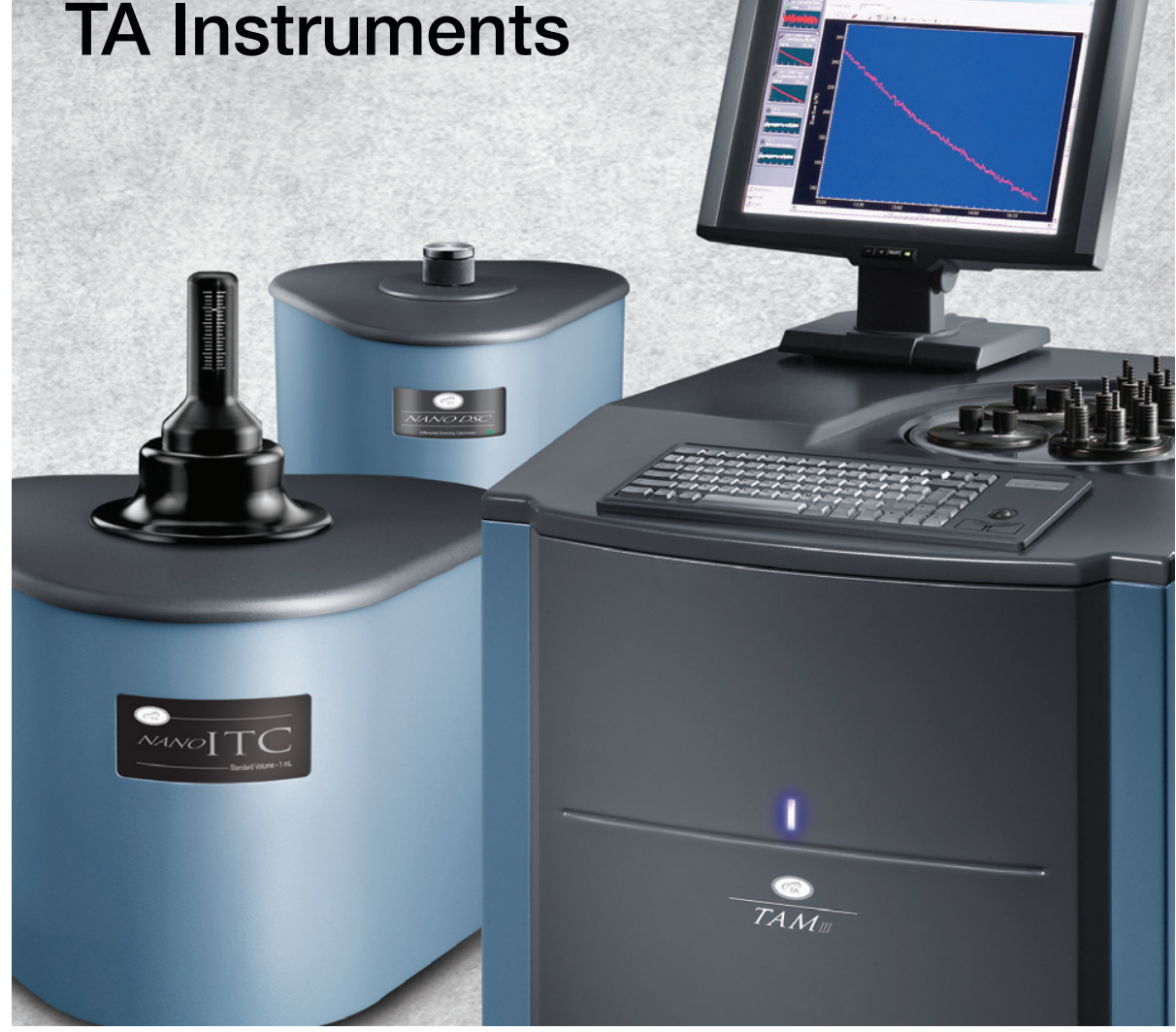

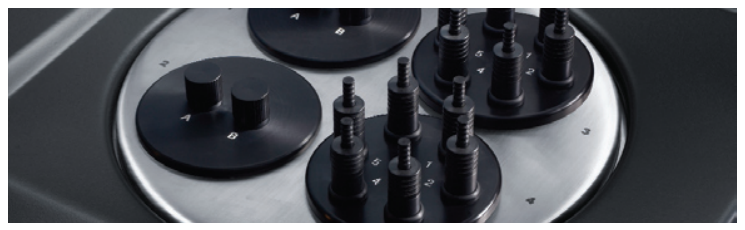
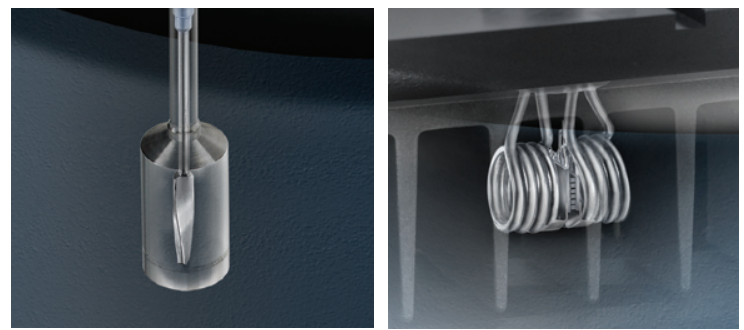

NANO DSC

- Highest Sensitivity Scanning Calorimeter

- Protein Denaturation, Stability

\section{NANO ITC}

- Highest Sensitivity Titration Calorimeter

- Protein Binding, Interactions, Kinetics

\section{TAM III}

- Highest Sensitivity Isothermal Calorimeter

- Stability, Compatibility, Morphology 